\title{
Diagnóstieo de enfermagem segundo a taxonomia II da NANDA de pacientes internados em uma unidade de terapia intensiva em Imperatriz, Maranhão, Brasil
}

\section{|Nursing Diagnosis by NANDA Taxonomy |l of Patients in Intensive Care Unit of Imperatriz, Maranhao, Brazil}

\author{
Cláudia de Sousa Fidelis' \\ Ele Diana Lopes Moraes² \\ Maria Luiza Rego Bezerra ${ }^{3}$ \\ Rodson Glauber Ribeiro Chaves ${ }^{4}$ \\ Simony Fabiola Lopes Nunes ${ }^{5}$
}

\begin{abstract}
Resumo
O presente estudo teve como objetivo identificar os principais $D E$ segundo a Taxonomia II da Norte American Nurding Diagnoses Associativo (NANDA), apresentados pelos pacientes internados em uma Unidade de Terapia Intensiva (UTI). Estudo transversal descritivo, realizado na UTI do Hospital Municipal de Imperatriz, MA, com uma amostra intencional de 40 pacientes. Foi evidenciado um total de 21 DE, 10 pacientes apresentaram frequência $\geq 70 \%$, destacando-se o risco de infecção (100\%), integridade da pele prejudicada (100\%), ventilação espontânea prejudicada (95\%), débito cardíaco diminuído $(80 \%)$, mobilidade no leito prejudicada (75\%), troca de gases prejudicada $(82,5 \%)$, conforto prejudicado $(77,5 \%)$, interação social prejudicada $(75 \%)$, processos familiares interrompidos $(72,5 \%)$ e comunicação verbal prejudicada $(70 \%)$. Os DE mais frequentes estiveram relacionados aos domínios segurança/proteção e atividade/repouso, os quais apontam as necessidades fisiológicas, como as mais comuns da amostra estudada. O uso dos DE se torna importante na rotina de trabalho de uma UTI, pois eles têm a função de guiar e justificar as intervenções de enfermagem. Diante do exposto, percebe-se a relevância da identificação dos DE, pois a delimitação destes mostra-se imprescindível para uma assistência de qualidade fundamentada no conhecimento científico.
\end{abstract}

\section{Abstract}

This study aimed to identify the main nursing diagnosis according to Taxonomy II Nort American Nursing Diagnosis Association (NANDA), presented by patients admitted to an Intensive Care Unit (ICU). Was performed in the ICU - Hospital Municipal de Imperatriz - MA, with a purposive sample of 40 patients. We found a total of 21 nursing diagnosis. Of these, 10 had a frequency $\geq 70 \%$, highlighting risk for infection $(100 \%)$, impaired skin integrity $(100 \%)$, impaired spontaneous ventilation (95\%), decreased cardiac output (80\%), bed mobility impaired (75\%), impaired gas exchange (82.5\%), impaired comfort (77.5\%), impaired social interaction $(75 \%)$, family processes $(72.5 \%)$ and impaired verbal communication $(70 \%)$. The most frequent nursing diagnosis domains were related to security / protection and activity / rest, which denote the physiological needs as the most common sample. The use of nursing diagnosis becomes important in the routine work of an ICU because it serves to guide and justify nursing interventions. Given the above, one realizes the importance of identifying the nursing diagnosis, because these boundaries, appear essential for quality care based on scientific knowledge.
Deseritores: unidades de terapia intensiva, euidados de enfermagem, diagnóstico de enfermagem.

Keywords: intensive care units. nursing care. nursing diagnosis.

${ }^{1}$ Enfermeira. Universidade Federal do Maranhão - Centro de Ciêneias Sociais, Saúde o Tecnologia.

${ }^{2}$ Enfermeira. Universidade Federal do Maranhão - Centro de Ciêneias Sociais, Saúde e Teenologia

${ }^{3}$ Enfermeira. Especialista. Professora do Curso de Enfermagem da Universidade Federal do Maranhão - Centro de Ciêneias Sociais, Saúde e Teenologia

${ }^{4}$ Enfermeiro. Especialista em Saúde Públiea. Professor do Curso de Enfermagem da Universidade Federal do Maranhão - Centro de Ciêneias Sociais, Saúde e Tecnologia.

5 Enfermeira. Especialista. Professora do Curso de Enfermagem da Universidade Federal do Maranhão - Centro de Ciêneias Sociais, Saúde e Teenologia.

Para correspondência:

Rodson Glauber Ribeiro Chaves

E-mail: rodson_ribeiro8@hotmail.com 
Introdução

A Unidade de Terapia Intensiva (UTI) é o setor destinado ao tratamento de pacientes em estado crítico, dispõe de uma infraestrutura própria, recursos materiais específicos e recursos humanos especializados, que através de uma prática assistencial segura e contínua busca o reestabelecimento das funções vitais do corpo que se encontram afetadas. ${ }^{1}$

O paciente é considerado crítico quando apresenta instabilidade de um ou mais órgãos vitais ou encontra-se na iminência de apresentar alguma alteração hemodinâmica, ou seja, é aquele que sofreu uma ameaça vital em seus parâmetros fisiológicos, em seu meio interno, sendo potencialmente reversível. ${ }^{2}$

Nesse sentido, a enfermagem tem forte influência na recuperação do estado de saúde e manutenção do equilíbrio do paciente, havendo estreita relação entre as ações de enfermagem e a prevenção de outros agravos. ${ }^{3}$

Para organizar esse cuidado ao paciente crítico, a enfermagem utiliza como estratégia o processo de enfermagem (PE), ferramenta que assegura uma prática assistencial adequada e individualizada ao paciente. Esse desenvolvimento da assistência de enfermagem humanizada, crítica e holística por meio do PE só são alcançados através do desenvolvimento de suas etapas: histórico de enfermagem, diagnóstico de enfermagem (DE), intervenção de enfermagem, implementação e avaliação de enfermagem. ${ }^{4,5}$

A preocupação com a necessidade de uma padronização da linguagem para definir os conhecimentos específicos da enfermagem, ganhou dimensões significativas na década de 70, na América do Norte. Assim, pensando nessa padronização e na necessidade de se desenvolver uma terminologia para descrever os problemas de saúde diagnosticados e tratados por enfermeiros, em 1973, foi realizada a $1^{\mathrm{a}}$ Conferência Nacional sobre Classificação dos Diagnósticos de Enfermagem. 5,6

Em 1982, aconteceu $5^{\text {a }}$ Conferência Nacional sobre Classificação dos DE e foi apresentada uma lista de $50 \mathrm{DE}$, sendo essa aceita para testes clínicos e que posteriormente originou a criação da North American Nursing Diagnosis Association (NANDA).5,7

A NANDA é um sistema de classificação que organiza os DE com a finalidade de promover sua reflexão e compreensão. Ao contrário do Código Internacional de Doenças (CID), que procura descrever uma doença, a taxonomia dos diagnósticos de enfermagem NANDA preocupa-se em identificar a situação de saúde/doença dos indivíduos internados, resultando em um cuidado de enfermagem individual e integral fundamentado no conhecimento científico de descrever a reação do paciente diante da doença. 1,8,9

A primeira taxonomia da NANDA foi aprovada em 1986, sendo organizada em noves padrões de resposta da pessoa (trocar, comunicar, relacionar, valorizar, escolher, mover, perceber, conhecer, sentir). ${ }^{5}$ Em 2001, foi publicada a taxonomia II, caracterizando-se por ser multiaxial e com flexibilidade da nomenclatura, o que permitiu modificações e inclusão de novos diagnósticos. ${ }^{10}$

O DE representa uma das mais importantes fontes de conhecimento científico específico de enfermagem, fornecendo critérios mensuráveis para avaliação da assistência, além de direcionar o cuidado, facilitar a pesquisa e o ensino, estimular o paciente a participar de seu tratamento e do plano terapêutico, visa também contribuir para expansão dos conhecimentos próprios da enfermagem. ${ }^{11}$

Desta forma, um DE representa um julgamento clínico sobre as respostas do indivíduo, da família ou da comunidade a problemas de saúde/processos vitais reais ou potenciais. Constitui a base para a seleção de intervenções de enfermagem e para o alcance dos resultados nessa área, os quais o enfermeiro é responsável. ${ }^{9}$

Em um ambiente de terapia intensiva, as necessidades de cuidados de enfermagem são mais complexas, pois os pacientes exigem avaliações críticas, rápidas e com planos de cuidados abrangentes. $^{12}$ Portanto, este estudo faz-se necessário pelo fato dos DE apresentarem-se como ferramenta válida na qualidade da assistência de enfermagem, bem como na promoção de um cuidado seguro e eficaz para o paciente.

Assim, o presente estudo objetivou identificar os principais DE segundo a taxonomia II da NANDA apresentados pelos pacientes internados em uma UTI de Imperatriz - MA.

\section{Materiais e Método}

Trata-se de um estudo transversal descritivo, com abordagem quantitativa. $\mathrm{O}$ método descritivo caracteriza-se pelo emprego da quantificação tanto nas modalidades de coleta de informações, quanto no tratamento delas por meio 
de técnicas estatísticas e valem-se de amostras amplas e de informações numéricas. ${ }^{13}$ Os estudos de delineamento transversal tem como principal característica a coleta de dados em determinado ponto do tempo. ${ }^{14}$

O presente estudo foi realizado na UTI de um hospital da rede pública do município de Imperatriz, no estado do Maranhão. O hospital é referência na região Tocantina no atendimento em nível secundário de atenção à saúde, nos serviços de urgência e emergência ao paciente adulto, tanto em situações clínicas como cirúrgicas.

A amostragem foi intencional, considerou-se todos os pacientes internados na UTI durante o período de coleta (março a junho de 2012), sendo que foram estabelecidos os seguintes critérios de inclusão: ter idade $\geq 18$ anos, estar internado a mais de 24 horas na UTI e nos casos de pacientes inconscientes deveria apresentar familiar ou responsável legal. Os pacientes com faixa etária inferior a 18 anos, com menos de 24 horas de internação e readmissões foram excluídos do estudo.

Dos 50 pacientes que foram internados a UTI durante o período de coleta, apenas 40 contemplaram os critérios de inclusão.

A coleta de dados foi realizada no período de março a junho de 2012, utilizando um instrumento semiestruturado de histórico de enfermagem, contendo anamnese e exame físico, fundamentado no modelo conceitual de Wanda de Aguiar Horta. ${ }^{15} \mathrm{O}$ histórico de enfermagem foi adaptado em forma de entrevista padronizada e estruturada, na qual foram abordados aspectos relacionados à identificação, queixa principal, história da doença atual, doenças pessoais pregressas, doenças familiares, hábitos de vida e sociais, e dados do prontuário, relacionados a informações clínicas e ambientais. Nos casos de pacientes com comprometimento de comunicação, a fonte de informação foi o familiar direto e/ou responsável.

Para análise e síntese dos dados iniciou-se a formulação dos diagnósticos de enfermagem de acordo com a taxonomia II da NANDA 2012-2014. ${ }^{9}$ Após a categorização dos mesmos, estes foram organizados e agrupados de maneira lógica e sistematizada, para assim serem validados por enfermeiros docentes especialistas na área.

A digitação dos dados foi realizada no Programa Epi Info, versão 3.5.1 (CDC: http:// http://wwwn.cdc.gov/epiinfo/3.5/), que posteriormente foram organizados e apresentados em tabelas na forma de frequência e percentagem. Em seguida, utilizou-se a técnica de Delphi,16 os DE com frequência $\geq 70 \%$ foram listados de acordo com o domínio a que pertencem.

A pesquisa respeitou os preceitos éticos e legais a serem seguidos nas investigações envolvendo seres humanos, conforme preconiza a Resolução 196/96, do Conselho Nacional de Saúde. ${ }^{17}$ Os participantes do estudo após o esclarecimento sobre os objetivos e a forma de condução da pesquisa concordaram em participar, assinando o termo de consentimento livre e esclarecido (TCLE). Aqueles sem condições de assinar o TCLE, procedeu-se a obtenção junto aos familiares ou responsáveis legais.

O estudo foi aprovado pelo Comitê de Ética em Pesquisa do Hospital Universitário da Universidade Federal do Maranhão (parecer $\mathrm{n}^{\circ}$ 71997/2013 HUFMA).

\section{Resultados e Diseussão}

Da amostra composta por 40 pacientes, observou-se a prevalência de $65 \%$ do sexo masculino e $35 \%$ do sexo feminino. Dados semelhantes foram encontrados na pesquisa de Laizo et al. (2010) ${ }^{18}$, avaliou-se 85 prontuários de pacientes submetidos à cirurgia cardíaca (revascularização do miocárdio) na Santa Casa de Misericórdia de Juiz de Fora - MG (SCMJF), na UTI cirúrgica, no período de março a maio de 2009, dos 85 pacientes, 52 (61,17\%) eram do sexo masculino e $33(38,82 \%)$ do sexo feminino.

No presente estudo verificou-se que a faixa etária dos participantes variou entre 18 a 83 anos, sendo que $17,5 \%$ apresentaram idade entre 18 a 28 anos, 35\% tinham entre 29 a 39 anos e 47,5\% eram pacientes com idade de 40 anos ou mais. Estudos semelhantes, realizado em 2007, por Paganim et al.(2010) ${ }^{19}$ com uma amostra de 150 prontuários de um hospital público da serra gaúcha, mostra que a maioria dos pacientes também eram do sexo masculino $(60,7 \%)$ e outro estudo conduzido por Feijó et al. $(2006)^{20}$ com pacientes do Hospital Universitário da Federal do Ceará $(n=300)$ observou que o grupo etário característico de admissão no setor de terapia intensiva eram os pacientes com idade igual ou superior a 60 anos $(43 \%)$.

Na Tabela 1 são apresentados os principais domínios representativos e seus respectivos títulos diagnósticos, identificados nos pacientes do 
estudo, sendo descritos conforme a publicação atual da classificação dos diagnósticos de enfermagem da Taxonomia II da NANDA-I. Assim, verificou-se um total de 21 diagnósticos, sendo que 10 apresentaram frequência $\geq 70 \%$. procedimentos invasivos constituem risco para a presença de patógenos como as bactérias. O ambiente hospitalar também é um local que favorece o surgimento de patógenos, sendo assim, quanto maior o número de procedimentos invasivos, maior o risco de infecção. ${ }^{10}$

\begin{tabular}{llr}
\hline Domínio/título enfermagem & $f$ & \% \\
\hline Segurança/proteção & 40 & 100 \\
$\quad$ Risco de infecção & 40 & 100 \\
$\quad$ Integridade da pele prejudicada & & \\
Atividade/repouso & 38 & 95 \\
$\quad$ Ventilação espontânea prejudicada & 32 & 80 \\
$\quad$ Débito cardíaco diminuído & 30 & 75 \\
$\quad$ Mobilidade no leito prejudicada & 33 & 82,5 \\
Eliminação e troca & & \\
$\quad$ Troca de gases prejudicada & 31 & 77,5 \\
Conforto & & 75 \\
$\quad$ Conforto prejudicado & 30 & 72,5 \\
Papéis e relacionamentos & 29 & 70 \\
$\quad$ Interação social prejudicada & & 100 \\
$\quad$ Processos familiares interrompidos & 30 & \\
$\quad$ Cercepção/cognição & 40 & \\
$\quad$ Tomunicação verbal prejudicada & $4 I, ~$ &
\end{tabular}

Tabela 1. Frequência dos títulos diagnósticos de enfermagem identificados na UTI, descritos com seus respectivos domínios, de acordo com a Taxonomia II NANDA - I. Imperatriz - MA, 2013

Fonte: Elaboração própria

O domínio Segurança/proteção é definido como estar livre de perigo, lesão física ou dano ao sistema imunológico, conservação contra perdas e proteção da segurança e da ausência de perigos. ${ }^{9}$ No presente estudo, encontrou-se nesse domínio, os diagnósticos risco de infecção e integridade da pele prejudicada em todos os pacientes $(100 \%)$.

$\mathrm{O}$ risco de infeção diz respeito ao aumento do risco do paciente ser invadido por germes patogênicos. ${ }^{9}$ Assim, observou-se que o risco de infecção correspondeu as defesas primárias inadequadas (pele rompida, tecido traumatizado), doença crônica, procedimentos invasivos (cateterismo vesical, acesso venoso) e trauma.

Em estudo realizado por Salomé (2010) ${ }^{21}$, com 77 pacientes da UTI do Hospital Geral Vila Nova Cachoeirinha (HGVNC) do estado de São Paulo, foram encontrados dados semelhantes e verificou-se que o risco de infecção tem influência da diversidade de profissionais que lida diretamente com o paciente, a forma de assistência que é dispensada, o tempo de hospitalização, principalmente a quantidade e o tipo de procedimentos invasivos, visto que os
Quanto ao diagnóstico integridade da pele prejudicada (100\%) observou-se que na pesquisa, todos os participantes apresentaram este diagnóstico em decorrência da destruição de camadas da pele e rompimento da sua superfície. No estudo, os fatores relacionados à alteração da epiderme e/ou derme foram a idade, fatores mecânicos (pressão, contenção), imobilidade física e circulação prejudicada.

No estudo de Rocha et al. (2006) ${ }^{10}$, a pesquisa foi realizada em um hospital do município de Fortaleza- $\mathrm{CE}$, pertencente à rede pública e referência estadual, em nível terciário de atenção à saúde, na área de Cardiologia e Pneumologia, com uma amostra de 22 pacientes internados em UTI. O estudo mostra resultados semelhantes aos encontrados, o fator característico que se verifica entre esses pacientes críticos é que eles são restritos ao leito, possuem dificuldade de mobilização e entre os fatores que potencializam esse quadro, estão as situações clínicas de desidratação, estresse do setor e da internação e extremos de idade. ${ }^{22,23}$ 
No domínio Atividade/repouso, considera-se a produção, conservação, gasto ou equilíbrio de recursos energéticos ${ }^{9} \mathrm{e}$ os diagnósticos predominantes encontrados no estudo foram a ventilação espontânea prejudicada (95\%), o débito cardíaco diminuído $(80 \%)$ e a mobilidade no leito prejudicada (75\%).

Determina-se ventilação espontânea prejudicada quando há incapacidade do indivíduo em manter o padrão respiratório adequado. ${ }^{9}$ Assim, destaca-se como características definidoras encontradas entre os participantes: a dispneia, o uso da musculatura acessória e a frequência cardíaca aumentada, sendo que a fadiga da musculatura acessória, também esteve relacionada a esse diagnóstico.

O estudo realizado por Truppel et al. (2009) ${ }^{24}$ sobre a Sistematização da Assistência em Enfermagem em UTI Adultos de um Hospital de Ensino no estado do Paraná, no período de 1 de agosto a 30 de novembro de 2006 ( $n=6$ enfermeiros), evidenciou que 95\% dos pacientes examinados também apresentaram este diagnóstico.

O diagnóstico débito cardíaco diminuído é definido como quantidade insuficiente de sangue bombeado pelo coração para atender às demandas metabólicas corporais. ${ }^{9}$ No estudo, esse diagnóstico esteve relacionado à frequência cardíaca e ritmo alterados dos pacientes. Esses achados assemelham-se a outros estudos $12,18,21$, contudo, observa-se que as características que definem o diagnóstico nesses pacientes estão ligados a hipotensão, frequência cardíaca alterada, arritmias, pressão venosa central alterada, pressão do átrio esquerdo alterada, perfusão periférica prejudicada, alterações no aspecto e coloração da pele e oligúria. ${ }^{25}$

A Mobilidade no leito prejudicada $(75 \%)$ representa uma limitação aos movimentos independente de uma posição no leito para outra. 9,21 No presente estudo, esteve relacionada a força muscular insuficiente, dor e prejuízo neuromuscular.

A maioria dos pacientes internados em unidades intensivas tem dificuldade de deambulação no leito, capacidade prejudicada de mover-se de uma posição para outra e reposicionar-se na cama e cadeira, como consequência de diversos fatores: a entubação orotraqueal, queixas álgicas, por serem vítimas de traumas, ou, pelo uso contínuo de cateteres, drenos e demais dispositivos invasivos. ${ }^{21}$

No domínio Eliminação e troca considerou-se as secreções e excreções de produtos residuais do organismo, e no estudo teve destaque o diagnóstico de enfermagem troca de gases prejudicada $(82,5 \%)$, trata-se da incapacidade do indivíduo em manter a respiração espontânea. ${ }^{9}$ As alterações respiratórias constituem em um problema comum nos pacientes internados em UTI, tanto por motivo da internação como por complicação desta, tendo em vista o elevado número de procedimentos invasivos, propiciando o desenvolvimento de infecções que podem potencializar a gravidade da fisiologia do aparelho respiratório. ${ }^{3}$

O diagnóstico troca de gases prejudicada, também foi observado por Arruda e Garcia $(2000)^{27}$ em estudo realizado em um hospital público, do município de João Pessoa- PB com 22 pacientes adultos que estiveram internados na UTI, os dados mostraram que a equipe de assistência deve planejar as ações evitando as complicações mais frequentes dos pacientes que necessitam de cuidados intensivos, como por exemplo as alterações no padrão e função ventilatória.

No domínio Conforto o diagnóstico conforto prejudicado $(77,5 \%)$, definido como falta percebida de sensação de conforto, alívio, transcendência nas dimensões física, psicoespiritual, ambiental e social. ${ }^{9}$ No presente estudo foi observado em seis características definidoras: o choro, lamentação, falta de privacidade, padrão de sono perturbado e relatos de sentir frio. Corroborando com esses achados, diagnóstico semelhante esteve presente em 3,8\% dos participantes do estudo de Carvalho et al. $(2008)^{26}$ realizado com 26 pacientes adultos internados na UTI de um hospital de ensino, de grande porte, do interior paulista sobre relações entre as etapas do processo de enfermagem em pacientes críticos.

No domínio Papéis e relacionamentos destacou-se interação social prejudicada $(75 \%)$ e processos familiares interrompidos $(72,5 \%)$ que podem ser definidos como quantidade insuficiente, ou qualidade ineficaz de troca social, mudança nos relacionamentos e/ou no funcionamento da família. Relacionado à ausência de pessoas significativas, barreiras de comunicação e isolamento terapêutico. Estudo realizado por Silva et al. $(2011)^{28}$, com oito pacientes internados em uma UTI no estado de Rondônia mostra divergência quanto a interação social (38\%) e processos familiares interrompidos (13\%).

No domínio Percepção/cognição, o diagnóstico comunicação verbal prejudicada, definido como habilidade diminuída, retardada ou ausente para receber, processar, transmitir e usar um sistema de símbolos ${ }^{9}$ ocorreu em $70 \%$ dos 
pacientes, esse diagnóstico esteve relacionado com a traqueostomia, tubo orotraqueal, ventilação mecânica e dispneia acentuada.

No estudo realizado por Salomé $(2010)^{21}$ esse diagnóstico também esteve presente, o que evidenciou a dificuldade da verbalização dos pacientes e sua relação direta com a entubação orotraqueal, retardo da entubação e fatores relacionados à dificuldade respiratória, em destaque a dispneia e a taquipneia.

Assim, diante dos diagnósticos de enfermagem encontrados no presente estudo, o conhecimento dos problemas de saúde de um grupo de pacientes com características comuns como esse que direciona a assistência de enfermagem, fornece subsídios para a elaboração de um plano de cuidados e implementação de intervenções de enfermagem.

\section{Conelusões}

No presente estudo foi evidenciado um total de 21 diagnósticos de enfermagem. Dentre estes, 10 apresentaram frequência $\geq 70 \%$, com destaque para risco de infecção $(100 \%)$, integridade da pele prejudicada (100\%), ventilação espontânea prejudicada (95\%), troca de gases prejudicada $(82,5 \%)$, débito cardíaco diminuído $(80 \%)$, conforto prejudicado $(77,5 \%)$, mobilidade no leito prejudicada (75\%), interação social prejudicada $(75 \%)$, processos familiares interrompidos $(72,5 \%)$ e comunicação verbal prejudicada (70\%). Os DE mais frequentes estiveram relacionados aos domínios segurança/proteção, eliminação/troca e atividade/repouso, os quais denotam as necessidades fisiológicas como as mais comuns encontradas na amostra estudada.

Observa-se que a construção de um conceito diagnóstico de enfermagem se torna importante na rotina de cuidado em UTI, pois ele tem a função de guiar e justificar as intervenções de enfermagem, com uma linguagem reconhecida, o que torna essas intervenções viáveis, além de direcionar a assistência de enfermagem para as necessidades de cada paciente, facilitando a escolha de intervenções adequadas, no sentido de registrar de forma objetiva as reações do paciente e permitir a subsequente avaliação dos cuidados de enfermagem implementados.

Assim, a identificação dos diagnósticos é fundamental para os enfermeiros, pois os permite propor intervenções fundamentadas e específicas que proporcionem a implementação de ações eficazes em um período curto de tempo para a resolução dos problemas identificados com uma assistência de qualidade fundamentada no conhecimento científico. A partir dos achados, aponta-se a necessidade de realizar outros estudos voltados para a percepção de DE segundo a Taxonomia II da NANDA- I em pacientes internados em Unidade de Terapia Intensiva.

\section{Referências}

1. Amante LN, Rossetto AP, Schneider DG. Sistematização da assistência de enfermagem em unidade de terapia intensiva sustentada pela teoria de Wanda Horta. Rev. Esc. Enferm. USP 2009; 43(1): 54-64.

2. Brasil. Ministério da Saúde. Portaria n ${ }^{\circ} 3432$, de 12 de agosto de 1998. Estabelece critérios de classificação para as Unidades de Tratamento Intensivo - UTI. Brasília: Ministério da Saúde, 1998.

3. Benedet AS, Brasil N. Sistematização da assistência de enfermagem e as necessidades de cuidados de pacientes internados em terapia intensiva. Gestão \& Saúde 2012; 03(02): 800-815.

4. Silva, DG, Freiberger MF, Coelho MPPM, Chocair DAF, Silva SAM. A identificação de diagnósticos de enfermagem da taxonomia II de NANDA em uma unidade de terapia. Rev Cientifica Faema 2011; 02(1): 42-54.

5. Chaves LD. Sistematização da Assistência de Enfermagem: considerações teóricas e aplicabilidade. São Paulo: Martinari, 2009.

6. Cruz DALM. Sistema de assistência de enfermagem: evolução e tendências. 4. ed. São Paulo: Icone, 2008.

7. Herdman T. Diagnósticos de enfermagem da NANDA: definições e classificação 2012-2014. Porto Alegre: Artmed, 2012.

8. Mendes MA. Sistematização da Assistência de Enfermagem Usando Raciocínio Baseado em Casos Implementado em JAVA [dissertação]. Ribeirão Preto (SP): Escola Politécnica de Ribeirão Preto Universidade de São Paulo; 2009.

9. North American Nursing Diagnosis Association (NANDA). Diagnósticos de enfermagem da NANDA: definições e classificações 2012-2014. Tradução de Jeanne Liliane Marlene Michel. Porto Alegre (RS): Artmed, 2013.

10. Rocha LA, Maia TF, Silva LF. Diagnósticos de enfermagem em pacientes submetidos à cirurgia cardíaca. Rev Bras Enferm 2006; 59(3): 321-32.

11. Galdeano LE, Rossi LA, Pezzuto, TM. Diagnósticos de enfermagem de pacientes no período préoperatório de cirurgia cardíaca. Rev. esc. enferm. USP 2004; 38(03): 307-16.

12. Bulechek G, Butcher, H, Dochterman, J, Wagner, C. (2012). Nursing interventions classification (NIC), 6th edition. MO: Mosby.

13. Marconi MA, Lakatos EM. Metodologia Científica. 5. ed. São Paulo: Atlas, 2007.

14. Polit DF, Beck CT, Hungler BP. Fundamentos da Pesquisa em Enfermagem: métodos, avaliação e utilização. 5. ed. Porto Alegre: Artmed, 2004.

15. Horta WA. O processo de enfermagem. São Paulo: EPU, 1979. 
16. Keeney S, Hasson F, Mckenna H. Consulting the oracle: ten lessons from the Delphi Technique in nursing research. J Adv Nurs 2006; 2(53): 205-12.

17. Brasil. Ministério da Saúde. Conselho Nacional de Saúde. Resolução 196/1996. Brasília: Ministério da Saúde, 1996.

18. Laizo A, Delgado FEF, Rocha GM. Complicações que aumentam o tempo de

permanência na unidade de terapia intensiva na cirurgia cardíaca. Rev Bras Cir Cardiovasc 2010; 25(2): 166-171.

19. Paganin A., Menegat P, Klafke T, Lazzarotto A, Fachinelli TS, Chaves IC, Souza EM. Implantação do diagnóstico de enfermagem em unidade de terapia intensiva: uma análise periódica. Rev. Gaúcha Enferm 2010; 31(02): 30713.

20. Feijó CAR, Leite JFO, Martins ACS, Furtado JAH, Cruz LLS, Meneses FA. Gravidade dos pacientes admitidos à unidade de terapia intensiva de um Hospital Universitário Brasileiro. Rev. bras. ter. intensiva 2006; 18 (01): 18-21.

21. Salomé GM. Diagnósticos de enfermagem dos pacientes internados em uma unidade de terapia intensiva. Saúde Coletiva 2010; 08(47): 24-28.

22. Araújo TM, Araújo MFM, Caetano JÁ, Galvão MTG, Damasceno MMC. Diagnósticos de enfermagem para pacientes em risco de desenvolver úlcera por pressão. Rev. bras. enferm 2011; 64(4): 671-676.
23. Oliveira HAD, Vieira OS, Cruz I. Como eu cuido de integridade da pele prejudicada na UTI: Estudo de caso. Rio de Janeiro: UFF; 2005.

24. Truppel, TC, Meier MJ, Calixto RC, Peruzzo SA; Crozeta K. Sistematização da assistência de enfermagem em unidade de terapia intensiva. Rev. Bras. Enferm 2009; 62(2): 221-7.

25. Pivoto FL Filho WDL, Santos SSC, Almeida MA, Silveiras RS. Diagnósticos de enfermagem em pacientes no pós-operatório de cirurgias cardíacas. Acta. Paul. Enferm 2010; 23(05): 665-70.

26. Carvalho EC, Martins FTM, Dalri MCB, Canini SRMS, Laus AM, Bachion MM, et al. Relações entre a coleta de dados, diagnósticos e prescrições de enfermagem a pacientes adultos de uma unidade de terapia intensiva. Rev. Latino-Am. Enfermagem 2008 16(4): 700-706.

27. Arruda AJCG, Garcia TR. Diagnósticos de enfermagem relacionados à oxigenação, atribuídos a vítimas de trauma admitidos em CTI. Rev Bras Enferm 2000; 53(3): 363-74.

28. Silva DG, Freiberger MF, Machado M P P, Chocair DFA, Menezes PS, Sâmia SAM. A identificação de diagnósticos de enfermagem da taxonomia II de nanda em uma unidade de terapia intensiva. Rev Cie Fac Edu Mei Amb 2011; 2(1):42-54. 